\title{
The Pediatric Quality of Life Inventory ${ }^{\mathrm{TM}}$ (PedsQL ${ }^{\mathrm{TM}}$ ) family impact module: reliability and validity of the Brazilian version Ana C Scarpelli1 ${ }^{1}$, Saul M Paiva*1,2, Isabela A Pordeus ${ }^{1}$, James W Varni ${ }^{3}$, Cláudia M Viegas ${ }^{1}$ and Paul J Allison ${ }^{2}$
}

\author{
Address: ${ }^{1}$ Department of Pediatric Dentistry and Orthodontics, Faculty of Dentistry, Federal University of Minas Gerais - Av. Antônio Carlos 6627, \\ Belo Horizonte, MG, 31270-901, Brazil, 2Faculty of Dentistry, McGill University, 3640 University Street, Montreal, QC, H3A 2B2, Canada and \\ ${ }^{3}$ Department of Pediatrics, College of Medicine, Department of Landscape Architecture and Urban Planning, College of Architecture, Texas A\&M \\ University, 3137 TAMU - College Station, TX, 77843-3137, USA \\ Email: Ana C Scarpelli - anascarp@yahoo.com.br; Saul M Paiva* - saul.paiva@mcgill.ca; Isabela A Pordeus - isabela@netuno.lcc.ufmg.br; \\ James W Varni - jvarni@archmail.tamu.edu; Cláudia M Viegas - claudiamviegas@yahoo.com.br; Paul J Allison - paul.allison@mcgill.ca \\ * Corresponding author
}

Published: 20 May 2008

Health and Quality of Life Outcomes 2008, 6:35 doi:10.1 186/1477-7525-6-35
Received: 5 November 2007

Accepted: 20 May 2008

This article is available from: http://www.hqlo.com/content/6/1/35

(c) 2008 Scarpelli et al; licensee BioMed Central Ltd.

This is an Open Access article distributed under the terms of the Creative Commons Attribution License (http://creativecommons.org/licenses/by/2.0), which permits unrestricted use, distribution, and reproduction in any medium, provided the original work is properly cited.

\begin{abstract}
Background: Pediatric health-related quality of life (HRQOL) has emerged as an important health outcome in clinical trials and healthcare research, for which HRQOL assessment instruments have played an important role. However, these instruments are not available in all countries or all languages. The Pediatric Quality of Life Inventory ${ }^{\mathrm{TM}}$ (PedsQL ${ }^{\mathrm{TM}}$ ) Family Impact Module is a multidimensional instrument developed to assess the impact of chronic medical conditions on the HRQOL of parents and family functioning. The objective of the present study was to evaluate the psychometric properties of the PedsQL ${ }^{\mathrm{TM}}$ Family Impact Module cross-culturally adapted for use in Brazil.
\end{abstract}

Methods: The PedsQL ${ }^{\mathrm{TM}}$ Family Impact Module was administered to 95 parents/guardians of children with cancer in active therapy from 2 to 18 years of age of both genders. Subjects were recruited by means of convenience samples from the Pediatric Hematology/Oncology Centers at two public hospitals. The 'in-patient' sample was defined as individuals who were hospitalized for the administration of chemotherapy. The 'out-patient' sample was defined as individuals who were receiving chemotherapy and were not hospitalized.

Results: Test-retest reliability exhibited correlation values ranging from $0.8 \mathrm{I}$ to 0.96 for all subscales. Internal consistency reliability was demonstrated for the PedsQL ${ }^{\mathrm{TM}}$ Family Impact Module: Total Scale Score $(\alpha=0.89)$, Parent Health-Related Quality of Life Summary Score $(\alpha=$ $0.83)$ and Family Summary Score $(\alpha=0.73)$. The Total Impact Score for the in-patient and outpatient samples was 67.60 and 56.43 , respectively $(p<0.01)$. The construct validity demonstrated that the PedsQL ${ }^{\text {TM }}$ Family Impact Module proved capable of distinguishing between families whose children/adolescents were hospitalized and families of children/adolescents who are being taken care of at home.

Conclusion: The Brazilian version of the Peds QL ${ }^{\mathrm{TM}}$ Family Impact Module was considered reliable and valid for assessing the impact of a chronic pediatric health condition on the HRQOL of parents and family functioning. The instrument should be field tested on other chronic pediatric illnesses. 


\section{Background}

Pediatric health-related quality of life (HRQOL) has emerged as an important health outcome in clinical trials and healthcare research. This is particularly true in the pediatric cancer population [1]. The significant progress in anti-neoplasm therapeutic protocols has enabled a reduction in mortality rates, especially in the last 40 years. Currently, many pediatric cancer patients can be cured if diagnosed and treated early. Therefore, there have been a growing number of studies aimed at assessing the HRQOL of pediatric cancer patients both during and following treatment. Decisions regarding the implementation of improvements in public healthcare may be adopted based on the impact of interventions on quality of life $[1,2]$.

The impact of disease and treatment on family functioning plays an important role in a child's adaptation to chronic disease. The family's capacity to cope with the multiple sources of stress and uncertainty associated with their child's diagnosis and treatment is likely to affect the child's quality of life. The functioning and well-being of parents/guardians depend on the child's situation as well. Information on the quality of life of pediatric cancer patients and their families allows the identification of families with special needs for support or psychological intervention $[3,4]$. There is a vast range of coping strategies displayed by families in relation to both practical and emotional difficulties [5]. Childhood cancer affects individuals between 0 and 18 years of age and represents from 0.5 to $3.0 \%$ of malignant tumors in most populations. In Brazil, the estimated incidence of children with tumors in 2006 was $2.5 \%$ (11,800 individuals) of all cases of malignant growth or tumors caused by abnormal, uncontrolled cell division (malignant neoplasm) [6]. A better understanding of children and their families while coping with this specific stressor could be valuable to healthcare professionals.

In order to assess the impact of childhood cancer on the HRQOL of families, the decision was made to use the Ped$\mathrm{sQL}^{\mathrm{TM}}$ Family Impact Module, which is a multidimensional instrument developed to assess the impact of chronic medical conditions on the HRQOL of parents and family functioning. The instrument was designed as a parent proxy-report instrument and can either stand alone or be integrated to the PedsQL ${ }^{\mathrm{TM}}$ Measurement Model $[7,8]$.

The objective of the current study was to evaluate the psychometric properties of the PedsQL ${ }^{\mathrm{TM}}$ Family Impact Module cross-culturally adapted to Brazilian Portuguese.

\section{Methods}

\section{Participants and Settings}

This validation study was developed in the city of Belo Horizonte, Minas Gerais, Brazil. Subjects were recruited from the Pediatric Hematology/Oncology Centers at two public hospitals. This convenience sample included 95 families of Brazilian children and adolescents between the ages of 2 and 18 years of both genders, with malignant neoplasm and receiving chemotherapy. Thus, we selected individuals who were receiving medical care to induce remission [1]. The existence of another illness or concomitant syndrome to the malignant neoplasm was established as an exclusion criterion.

Proxy-reports were filled out by 95 parents/guardians who were interviewed at the hospital units. 'In-patient' status was defined as individuals who were hospitalized for the administration of chemotherapy and were always accompanied by a family member. 'Out-patient' status was defined as individuals who only came to the hospital for the administration of chemotherapy and were being taken care of at home. Most of the patients were in out-patient treatment. The in-patient sample $(\mathrm{n}=29,30.5 \%)$ was interviewed while hospitalized and the out-patient sample $(\mathrm{n}=66,69.5 \%)$ was interviewed while awaiting medical care. During the interviews, the parents/guardians also responded to a form regarding information on their age, kinship and degree of schooling. Interviews were performed individually by one of the researchers (ACS) in a room specifically reserved for this end. The interviewer restricted herself to reading the questions and answers of the questionnaire. Data collection took place between August 1, 2006 and December 20, 2006. Prior to the interviews, approvals were obtained from the Human Research Ethics Committees of the institutions involved. Written informed consent terms were also obtained from the participants.

\section{Instrument}

The 36-item PedsQL ${ }^{\mathrm{TM}}$ Family Impact Module is a parentreport instrument designed to assess the impact of pediatric chronic health conditions on parents and the family. It includes 6 subscales measuring parents' self-reported functioning: Physical Functioning (6 items), Emotional Functioning (5 items), Social Functioning (4 items), Cognitive Functioning ( 5 items), Communication (3 items) and Worry (5 items); as well as 2 subscales measuring parent-reported family functioning: Daily Activities (3 items) and Family Relationships (5 items) [8]. The scale has five Likert response options, 'never', 'almost never', 'sometimes', 'often' and 'almost always' (corresponding to scores of 100, 75, 50, 25 and 0). Regarding the interpretation of the scale, higher scores indicate better functioning (less negative impact). The PedsQL ${ }^{\mathrm{TM}}$ Family Impact Module Total Scale Score is calculated as the sum of the 36 item scores divided by the number of items answered.

Two other scores can also be obtained. The Parent HRQOL Summary Score assesses the impact of cancer on 
the health-related quality of life of parents/guardians. The score is computed as the sum of the 20 item scores on the Physical, Emotional, Social and Cognitive Functioning Subscales divided by the number of items answered in these subscales.

The Family Functioning Summary Score assesses the impact of cancer specifically on family activities and relationships. The score is obtained from the sum of the 8 item scores on the Daily Activities and Family Relationships Subscales divided by the number of items answered in these subscales.

\section{Cross-cultural adaptation}

Linguistic validation of the PedsQL ${ }^{\mathrm{TM}}$ Family Impact Module was performed following the PedsQL ${ }^{\mathrm{Tm}}$ Measurement Model translation methodology $[9,10]$. The model of equivalence in the cultural adaptation of HRQOL instruments developed by Herdman et al. (1997) [9] was adopted for the planning, structuring and execution of the cross-cultural adaptation of the instrument [10].

The process was performed in five steps. In the first step, two translations from the original English-language instrument into Brazilian Portuguese were performed independently by two bilingual translators whose native language was Brazilian Portuguese.

In the second step, the two translated versions (T1 and T2) were analyzed by a group of specialists composed of 6 professionals from the field of Pediatric Oncology (one physician, three psychologists and two social workers). Special attention was given to the meaning of the words in the different languages (English and Portuguese) in order to obtain similar effects from respondents of different cultures. An effort was made to identify possible difficulties in understanding the questionnaire. A synthesisversion was developed (T3) as a result of this process.

The third step consisted of a backtranslation of the synthesis-version (T3) by a professional, bilingual translator whose native language was English. This translator had no access to the original instrument.

A subsequent comparison between the original version and the backtranslated version was performed by a third translator who was fluent in English and whose native language was Brazilian Portuguese. The fourth step was the analysis of semantic equivalence between the original and backtranslated questionnaires, assessed from the perspective of referential meaning of the constituent terms/ words and general meaning of each item $[9,11]$.

The concept of referential meaning was developed to evaluate the similarity between the literal meaning of the terms in the pairs of statements (original and backtranslated versions) $[9,11]$. Visual Analogue Scales were used for the analysis of referential meaning [12]. A single line with verbal and numeral descriptors at each end was constructed for each pair of statements (the original and adapted items). The Visual Analogue Scales were constructed with a horizontal line and vertical line anchors at either end labeled "complete meaning disagreement" and "complete meaning agreement", denoted as 0 and 100, respectively; the line was marked in units of 1 and labeled in units of 10 [13]. Thus, equivalence between the pairs of statements could be judged in a continuous form between 0 and $100 \%$.

The concept of general meaning was used to evaluate the similarity regarding the idea transmitted by the statements between original and backtranlated versions. [9,11] A qualitative evaluation was carried out to assess item equivalence between the two versions. Each pair of statements was classified as: unaltered, slightly altered, very altered and completely altered.

The fifth step involved a preliminary qualitative evaluation of the proposed synthesis version. The PedsQL ${ }^{\mathrm{TM}} \mathrm{Fam}$ ily Impact Module was then applied to 20 individuals. In this phase, the interviewer carried out cognitive debriefing interviews in which the interviewees had the opportunity to suggest changes in words, phrases and expressions. They could also suggest examples for clarifying the question and express opinions on the acceptability, relevance and ease of comprehension of the questionnaire.

\section{Statistical analysis}

Test-retest reliability was assessed using the Intraclass Correlation Coefficient (ICC) for total, summary and the 8 subscales scores. A 95\% confidence interval was adopted. The ICC was measured according to the following values: $\leq 0.40$ weak correlation; $0.41-0.60$ moderate correlation; 0.61-0.80 good correlation; and 0.81-1.00 excellent correlation $[14,15]$. The Weighted Kappa Coefficient $(\mathrm{kw})$ was also calculated for each question of the instrument to measure the degree of agreement for each pair of observations. The criteria considered in the interpretation of agreement: -1.0 to 0.0 poor; 0.0 to 0.20 discrete; 0.20 to 0.40 regular; 0.40 to 0.60 moderate; 0.60 to 0.80 substantial; 0.80 to 1.00 nearly perfect [16]. The PedsQL ${ }^{\mathrm{TM}}$ Family Impact Module was administered twice by the same researcher to 47 study participant families $(49.5 \%$ of the overall sample), with a 7 -day interval between occasions.

Internal consistency was determined using Cronbach's Alpha Coefficient. Values $\geq 0.70$ were considered acceptable for comparisons between groups $[17,18]$. 
Construct validity was established using the "knowngroups method". We hypothesized that families whose children/adolescents were hospitalized would report higher scores (less negative impact) than families whose children/adolescents were being taken care of at home. In Brazil, continuous health care in a hospital has a distinctive importance for families, with continuous access to physicians, nurses, medication and a balanced diet [19]. In order to determine the magnitude of the differences between families, effect sizes were calculated. This analysis was calculated by taking the difference between the inpatient mean and the out-patient mean divided by the pooled standard deviation. Effect sizes for differences in means are designated as small (0.20), medium (0.50) and large (0.80) [20].

Data analyses were carried out with SPSS statistical software (version 12.0).

\section{Results}

\section{Sample characteristics}

The instrument was applied to 95 parents/guardians of children/adolescents with malignant neoplasm; 66 (69.5\%) of the parents/guardians were related to individuals in the out-patient sample. Table 1 displays a descriptive analysis of the demographic characteristics of the overall sample. Most of the children/adolescents had been diagnosed with leukemia (55.8\%). The average age of the parents/guardians was 35.4 years (standard deviation = 9.7); $76.8 \%$ were mothers and $62.1 \%$ had up to 8 years of schooling.

In order to assess the test-retest reliability, the instrument were administered for a second time to 47 (49.5\%) of the 95 parents/guardians one week following the first interview. The health condition of the children was clinically similar between the first and the second interviews.

\section{Cross-cultural adaptation}

During the cross-cultural adaptation, the group of specialists stated that the concept of the impact of childhood cancer on the quality of life of the families used for development of the original instrument was pertinent to Brazilian culture. The assessment of semantic equivalence was performed between the items from the backtranslated synthesis-version and the items from the original version. Considering the referential meaning, $86.1 \%$ of the 36 items exhibited "complete meaning agreement", as rated on a Visual Analogue Scale. The general meaning remained unaltered in $86.1 \%$ of the pairs of statements. The interviewees reported that they enjoyed answering the questions and considered the research very important. The parents did not report any problems in understanding the instructions and response choices of the instrument.
Table I: Descriptive analysis: demographic characteristics of the sample

\begin{tabular}{|c|c|c|}
\hline \multirow[t]{2}{*}{ Demographic characteristics } & \multicolumn{2}{|c|}{ Total sample $(\mathrm{N}=95)$} \\
\hline & $\mathrm{N}$ & $\%$ \\
\hline \multicolumn{3}{|c|}{$\begin{array}{l}\text { Children/adolescent characteristics } \\
\text { Ages (years) }\end{array}$} \\
\hline $2-4$ & 33 & 34.8 \\
\hline $5-7$ & 16 & 16.8 \\
\hline $8-12$ & 28 & 29.5 \\
\hline $13-18$ & 18 & 19.0 \\
\hline \multicolumn{3}{|l|}{ Gender } \\
\hline Boys & 60 & 63.2 \\
\hline Girls & 35 & 36.8 \\
\hline \multicolumn{3}{|l|}{ Cancer diagnosis } \\
\hline Leukemia & 53 & 55.8 \\
\hline $\begin{array}{l}\text { Lymphoma and reticuloendothelial } \\
\text { neoplasm }\end{array}$ & 16 & 16.8 \\
\hline Others & 26 & 27.4 \\
\hline \multicolumn{3}{|l|}{ Groups } \\
\hline Out-patient & 66 & 69.5 \\
\hline In-patient & 29 & 30.5 \\
\hline \multicolumn{3}{|c|}{$\begin{array}{l}\text { Characteristics of parents/guardian } \\
\text { Ages (years) }\end{array}$} \\
\hline $20-40$ & 73 & 76.8 \\
\hline $41-79$ & 22 & 23.2 \\
\hline \multicolumn{3}{|l|}{ Relationship to patient } \\
\hline Mother & 73 & 76.8 \\
\hline Others & 22 & 23.2 \\
\hline \multicolumn{3}{|l|}{ Level of schooling } \\
\hline$\leq 8$ years & 59 & 62.1 \\
\hline$>8$ years & 36 & 37.9 \\
\hline
\end{tabular}

However, they made a number of suggestions for replacing words and expressions.

\section{Construct validity}

The construct validity of the PedsQL ${ }^{\mathrm{TM}}$ Family Impact Module was determined by comparing scores obtained by the parents/guardians from the in-patient and out-patient samples. Table 2 displays means, standard deviations, analysis of effect sizes and t-test results of the responses on each subscale of the PedsQL ${ }^{\mathrm{TM}}$ Family Impact Module in the in-patient and out-patient samples. The effect size ranged from medium to large for the all of the subscales except "Cognitive Functioning" and "Daily Activities".

\section{Reliability}

The test-retest reliability analysis of the PedsQL ${ }^{\mathrm{TM}}$ Family Impact Module scales is displayed in Table 3. The subscales exhibited excellent ICC values $(>0.80)$. Agreement of the items revealed Weighted Kappa Coefficient values of $0.31-0.85$, thereby ranging from regular to nearly perfect. 
Table 2: Scale descriptors for the PedsQL ${ }^{\mathrm{TM}}$ Family Impact Module: comparisons between in-patient and out-patient samples

\begin{tabular}{|c|c|c|c|c|c|c|c|c|c|}
\hline \multirow[b]{2}{*}{ Subscale } & \multirow[b]{2}{*}{ Number of items } & \multicolumn{3}{|c|}{ In-patient sample } & \multicolumn{3}{|c|}{ Out-patient sample } & \multirow[b]{2}{*}{ Difference } & \multirow[b]{2}{*}{ Effect Size } \\
\hline & & $\mathbf{N}$ & Mean & SD & $\mathbf{N}$ & Mean & SD & & \\
\hline Total Impact Score & 36 & 29 & 67.60 & 13.53 & 66 & 56.43 & 16.27 & $11.17^{* * * *}$ & 0.75 \\
\hline Parent HRQOL Summary Score & 20 & 29 & 72.20 & 13.86 & 66 & 62.18 & 17.07 & $10.02 * * *$ & 0.65 \\
\hline Physical Functioning & 6 & 29 & 70.55 & $20.4 I$ & 66 & 58.23 & 23.55 & $\left.12.3\right|^{* *}$ & 0.56 \\
\hline Emotional Functioning & 5 & 29 & 68.62 & 15.69 & 66 & 55.08 & 20.35 & $13.54^{* * * *}$ & 0.75 \\
\hline Social Functioning & 4 & 29 & 76.94 & 22.60 & 66 & 69.29 & 26.28 & 7.65 & 0.31 \\
\hline Cognitive Functioning & 5 & 29 & 74.48 & 25.72 & 66 & 68.79 & 22.21 & 5.69 & 0.24 \\
\hline Communication & 3 & 29 & 72.99 & 29.18 & 66 & 61.49 & 26.18 & 11.50 & 0.42 \\
\hline Worry & 5 & 29 & 48.28 & 26.74 & 66 & 33.18 & 19.68 & $15.09 * * * *$ & 0.65 \\
\hline Family Summary Score & 8 & 29 & 67.46 & 21.77 & 66 & 56.25 & 22.35 & $11.2 I^{*}$ & 0.51 \\
\hline Daily Activities & 3 & 29 & 48.85 & 35.55 & 66 & 49.50 & 32.15 & -0.64 & -0.02 \\
\hline Family Relationships & 5 & 29 & 79.31 & 23.89 & 66 & 59.24 & 26.86 & $20.07 * * *$ & 0.79 \\
\hline
\end{tabular}

$\mathrm{N}=$ number of individuals; $\mathrm{SD}=$ standard deviation.

$*_{p}<.05,{ }^{* *} \mathrm{p}<.02$, *** $\mathrm{p}<.01$; equal variance was not assumed for Social Functioning and Worry subscales.

Table 4 displays the internal consistency reliability alpha coefficients for PedsQL ${ }^{\mathrm{Tm}}$ Family Impact Module subscales. The Total Impact Scores, the Parent HRQOL Summary Score and the Family Summary Score achieved values greater than 0.70 in the total, in-patient and outpatient samples. However, some subscales presented values near or below 0.70 when assessed separately, the lowest (0.52) achieved on 'emotional functioning' subscale in the in-patient sample. The 'emotional functioning' and 'social functioning' subscales achieved Cronbach's alpha coefficients between 0.52 and 0.67 in the total, in-patient and out-patient samples.

The internal consistency reliability alpha coefficients for the Brazilian version and original English version of the PedsQL ${ }^{\mathrm{TM}}$ Family Impact Module are presented in Table 5. Both the original and Brazilian versions achieved Cronbach's alpha coefficients greater than 0.70 in the total, inpatient and out-patient samples.

\section{Discussion}

Care for children with cancer should encompass supporting and helping families to cope with all aspects of treatment, including the diagnosis and the uncertainty of the outcome. Psychological distress following a diagnosis of childhood cancer involves risks of long-term psychosocial problems for parents and families. High rates of depression or posttraumatic stress symptoms are reported. Frequently, the entire family experiences disruption in their daily routine. In order to help families adjust positively to the illness, the assessment of the heath-related quality of life of parents and families could contribute toward their healthcare needs $[3,21,22]$.
This study presents the reliability and validity of the Brazilian Portuguese version of the PedsQL ${ }^{\mathrm{TM}}$ Family Impact Module, a multidimensional instrument developed to assess the health-related quality of life (HRQOL) of parents and family in the context of childhood cancer. HRQOL measurements have been the target of research investigations in the healthcare field and a number of HRQOL assessment instruments have been developed. However, these instruments are not yet available in all countries or languages. Most questionnaires have been developed in English-speaking countries and adapted for use in other countries [23-25]. Considering the differences between social, cultural and economic aspects, the availability of cross-culturally valid, multi-lingual versions of instruments is important for obtaining reliable, comparable data [26].

The cross-cultural adaptation of the PedsQL ${ }^{\mathrm{TM}}$ Family Impact Module was performed following a specific protocol (PedsQL ${ }^{\mathrm{TM}}$ Measurement Model translation methodology) [27], which ensures the adoption of a single methodology for the adaptation of the scale in different countries. Regarding the assessment of the semantic aspects, it was concluded that the pairs of translation/ backtranslation statements achieved adequate equivalence vis-à-vis the original questionnaire. The involvement of the group of specialists should be emphasized, as they contributed with reflections and discussions, thereby promoting suitable adjustments in the developed synthesis-version.

Instruments should produce similar results in two or more administrations to the same individual, provided 
Table 3: PedsQL ${ }^{\mathrm{TM}}$ Family Impact Module: Test-retest reliability $(\mathbf{N}=47)$

\begin{tabular}{|c|c|}
\hline Subscale & Sample $(N=47)$ \\
\hline Total Impact Score & $0.96(0.92-0.97)^{*}$ \\
\hline Parent HRQOL Summary Score & $0.95(0.92-0.97) *$ \\
\hline Physical Functioning & $0.90(0.82-0.94) *$ \\
\hline Item I: ...tired during the day & $0.53 \#$ \\
\hline Item 2: ...tired in the morning & $0.55^{\#}$ \\
\hline Item 3: ...feel too tired to do things & $0.55 \#$ \\
\hline Item $4:$...headaches & $0.65 \#$ \\
\hline Item 5: ...body weakness & $0.60 \#$ \\
\hline Item 6: ...nausea & $0.77 \#$ \\
\hline Emotional Functioning & $0.81(0.66-0.90) *$ \\
\hline Item I: ...anxiety & $0.31 \#$ \\
\hline Item 2: ...sadness & $0.56 \#$ \\
\hline Item 3: ...angry & $0.54 \#$ \\
\hline Item 4: ...disappointment & $0.49 \#$ \\
\hline Item 5: ...helplessness and hopelessness & $0.65 \#$ \\
\hline Social Functioning & $0.93(0.87-0.96) *$ \\
\hline Item I: ...isolation & $0.68^{\#}$ \\
\hline Item 2: ...difficult to get help & $0.85 \#$ \\
\hline Item 3: ...difficult to find time to have fun & $0.60 \#$ \\
\hline Item $4:$...lack of energy to have fun & $0.61 \#$ \\
\hline Cognitive Functioning & $0.92(0.85-0.95) *$ \\
\hline Item I: ...difficult to pay attention to things & $0.63 \#$ \\
\hline Item 2: ...difficult to remember what people tell me & $0.46 \#$ \\
\hline Item 3: ...difficult to remember what I have just heard & $0.61 \#$ \\
\hline Item $4:$...difficult to think quickly & $0.60 \#$ \\
\hline Item 5: ...difficult to remember what I was just thinking & $0.64 \#$ \\
\hline Communication & $0.81(0.65-0.89) *$ \\
\hline Item I: ...people do not understand my family's situation & $0.45 \#$ \\
\hline Item 2: ...difficult to speak about my child's illness & $0.38 \#$ \\
\hline Item 3: ...difficult to tell the doctors and nurses how I feel & $0.6 I^{\#}$ \\
\hline Worry & $0.91(0.83-0.95) *$ \\
\hline Item I: ...worry whether my child's treatment is working & $0.66 \#$ \\
\hline Item 2: ...worry about the side effects of medications & $0.57 \#$ \\
\hline Item 3: ...worry about how people will react to the illness & $0.47^{\#}$ \\
\hline Item 4: ...worry about how the illness affects my family & $0.63 \#$ \\
\hline Item 5: ...worry about my child's future & $0.7 \mid \#$ \\
\hline Family Summary Score & $0.95(0.90-0.97) *$ \\
\hline Daily Activities & $0.89(0.80-0.94) *$ \\
\hline Item I: ...family activities takes more time and effort & $0.54 \#$ \\
\hline Item 2: ...difficult to find time to finish the household chores & $0.60 \#$ \\
\hline $\begin{array}{l}\text { Item 3: ...fatigue made it difficult to finish the household } \\
\text { chores }\end{array}$ & $0.64 \#$ \\
\hline Family Relationships & $0.85(0.73-0.92) *$ \\
\hline Item I: ...lack of communication between people in my family & $0.60 \#$ \\
\hline Item 2: ...conflicts between people in my family & $0.56 \#$ \\
\hline Item 3: ...difficult to make group decisions in my family & $0.69 \#$ \\
\hline Item 4: ...difficult to solve family problems & $0.73 \#$ \\
\hline Item 5: ...stress and tension between people in my family & $0.55 \#$ \\
\hline
\end{tabular}
$* p_{95} \leq 0.00$ I (2-tailed) Intraclass Correlation Coefficient (ICC) - Confidence Interval
(IC)

\#Weighted kappa Coefficient (kw) was calculated for each item

that the general clinical state has not been altered [24]. The analysis of test-retest reliability suggests the adequate stability of the PedsQL ${ }^{\mathrm{TM}}$ Family Impact Module. The 7day interval between interviews was important in diminishing the probability of systemic alterations in the clinical condition of the individuals. It is recommended that the interval between measurements be long enough to reduce the effects of memory and short enough to diminish the likelihood of systemic alterations. Although the definition of this interval is arbitrary, a period of 2 to 14 days is considered adequate [12,24,28,29].

Considering the internal consistency of the PedsQL ${ }^{\mathrm{TM}}$ Family Impact Module, the Cronbach's $\alpha$ coefficients exceeded the recommended minimum of 0.70 for the total impact score and the summary scores, demonstrating the adequate homogeneity of the scale. As in the original version, the Brazilian version performed reliably. However, values were heterogeneous when assessing each subscale separately. The 'emotional functioning', 'social functioning' and 'communication' subscales should be used disjointedly only for descriptive or exploratory analyses, as they did not achieve a alpha coefficient of 0.70 in the total sample. A study carried out in San Diego and Los Angeles (USA) with 339 families of individuals with cancer between the ages of 2 and 18 years found alpha coefficients of less than 0.70 in various subscales of the versions designed for children/adolescents [30]. This low internal consistency may be related to the small number of items that compose the subscales as well as the small sample size [30,31]. Furthermore, alpha coefficient values may be influenced by the low level of schooling in the sample [25,32].

Construct validity was evaluated using the differentiation of groups that are known to be distinct $[1,25,33,34]$. The data demonstrated statistically significant differences between families whose children/adolescents are hospitalized and families of children/adolescents who were being taken care of at home. The hypothesis established was supported: families whose individuals are hospitalized have higher functioning than those whose children are living at home. Therefore, the occurrence of illness implied limitations and difficulties in the functioning of the entire family. This fact was also reported in a study with 23 medically fragile pediatric patients in San Diego, United States [8].

Except for the "Daily Activities" subscale, the means obtained in all other subscales were greater in the inpatient sample, confirming that childhood cancer in hospitalized individuals had a lesser negative impact on family functioning than in those living at home $[8,21]$.

This study has certain limitations that should be recognized. The generalizability of the findings is limited by two factors: the small sample size and the selection of a specific chronic pediatric condition. Sample size is an ever present difficulty in studies on individuals afflicted with cancer, stemming from the low prevalence of the illness $[25,29,32,35]$. In order to broaden this convenience sample, the study encompassed the two largest childhood cancer treatment hospitals in Belo Horizonte, Brazil. Although the sample size decreased the probability of 


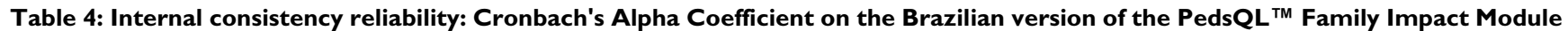
for total, in-patient and out-patient samples

\begin{tabular}{lccc}
\hline \multicolumn{1}{c}{ Subscale } & Total Sample (N = 95) & In-patient sample (N = 29) & Out-patient sample (N = 66) \\
\hline Total Impact Score & 0.89 & 0.86 & 0.89 \\
Parent HRQOL Summary Score & 0.83 & 0.78 & 0.83 \\
Physical Functioning & 0.70 & 0.64 & 0.69 \\
Emotional Functioning & 0.62 & 0.52 & 0.59 \\
Social Functioning & 0.65 & 0.61 & 0.67 \\
Cognitive Functioning & 0.75 & 0.84 & 0.69 \\
Communication & 0.60 & 0.72 & 0.52 \\
Worry & 0.70 & 0.78 & 0.58 \\
& & & \\
Family Summary Score & 0.73 & 0.76 & 0.70 \\
Daily Activities & 0.69 & 0.77 & 0.66 \\
Family Relationships & 0.81 & 0.81 & 0.78
\end{tabular}

detecting significant differences, 7 of 11 comparisons between in-patient and out-patient samples were statistically significant regarding the different scales and subscales. Further studies should be conducted to test the performance of the instrument on groups of children with other chronic health conditions. It should also be stressed that the scale was developed to be self-administered. However, due to the low level of schooling among the individuals of the study, the option was made to administer the questionnaire in interview form in all cases. A number of studies have demonstrated that the mode of administration does not affect the performance of the instruments [25,33-37]. Nevertheless, a comparison between the interview mode of administration and selffilled out mode of administration needs further investigation. In the present study, there was no report by the parents/guardians of any lack of comprehension regarding the questions.

\section{Conclusion}

The Brazilian version of the PedsQL ${ }^{\mathrm{Tm}}$ Family Impact Module exhibited adequate properties regarding the reliability and validity of the construct. This suggests its usefulness as a parameter in studies assessing the impact of pediatric cancer on the HRQOL of parents and family functioning. The PedsQL ${ }^{\mathrm{Tm}}$ Family Impact Module should be field tested on other chronic pediatric illnesses in order to permit the generalization of the findings.

\section{Abbreviations}

PedsQL ${ }^{\mathrm{Tm}}$ : Pediatric Quality of Life Inventory ${ }^{\mathrm{Tm}}$; HRQOL: Health-Related Quality of life; ICC: Intraclass Correlation Coefficient.

\section{Competing interests}

The authors declare that they have no competing interests.

Table 5: Internal consistency reliability: Cronbach's alpha coefficient on the Brazilian and original versions of the PedsQL ${ }^{\mathrm{TM}}$ Family Impact Module for total, in-patient and out-patient samples

\begin{tabular}{|c|c|c|c|c|}
\hline \multirow[b]{2}{*}{ Scale } & \multirow[b]{2}{*}{ Items $^{\mathbf{a}}$} & \multicolumn{3}{|c|}{ Cronbach's $\alpha$ coefficient } \\
\hline & & Total sample & In-patient sample & Out-patient sample \\
\hline \multicolumn{5}{|c|}{ Total Impact Score } \\
\hline Brazilian version & 36 & 0.89 & 0.86 & 0.89 \\
\hline Original Version & 36 & 0.97 & 0.97 & 0.95 \\
\hline \multicolumn{5}{|c|}{ Parent HRQOL Summary Score } \\
\hline Brazilian version & 20 & 0.83 & 0.78 & 0.83 \\
\hline Original Version & 20 & 0.96 & 0.96 & 0.95 \\
\hline \multicolumn{5}{|c|}{ Family Summary Score } \\
\hline Brazilian version & 8 & 0.73 & 0.76 & 0.70 \\
\hline Original Version & 8 & 0.90 & 0.93 & 0.89 \\
\hline
\end{tabular}




\section{Authors' contributions}

ACS, SMP, IAP, JWV and PJA conceptualized the rationale and design of the study, ACS and CMV performed the statistical analysis and interpretation of the data, ACS, SMP and PJA drafted the manuscript. All authors read and approved the final manuscript.

\section{Acknowledgements}

This research was supported by National Council for Scientific and Technological Development (CNPq), Ministry of Science and Technology, Brazil.

\section{References}

I. Varni JW, Katz ER, Seid M, Quiggins DJ, Friedman-Bender A: The pediatric cancer quality of life inventory-32 (PCQL-32): I. Reliability and validity. Cancer 1998, 82(6): I I84- I I96.

2. Mulhern RK, Palmer SL: Neurocognitive late effects in pediatric cancer. Curr Probl Cancer 2003, 27(4): I77-197.

3. Goldbeck L: The impact of newly diagnosed chronic paediatric conditions on parental quality of life. Qual Life Res 2006, I5(7): I| $\mid$ |-| | $3 \mid$.

4. Wallander JL, Varni JW: Effects of pediatric chronic physical disorders on child and family adjustment. Journal of child psychology and psychiatry, and allied disciplines 1998, 39(I):29-46.

5. Eiser C: Psychological effects of chronic disease. Journal of child psychology and psychiatry, and allied disciplines 1990, 3 I (I):85-98.

6. Registro de Cancer de Base Populacional [http://
[ www.inca.gov.br/conteudo view.asp?id=353]

7. Varni JW, Seid M, Rode CA: The PedsQL: measurement model for the pediatric quality of life inventory. Medical care 1999 , 37(2): I26-139.

8. Varni JW, Sherman SA, Burwinkle TM, Dickinson PE, Dixon P: The PedsQL Family Impact Module: preliminary reliability and validity. Health and quality of life outcomes 2004, 2:55.

9. Herdman M, Fox-Rushby J, Badia X: 'Equivalence' and the translation and adaptation of health-related quality of life questionnaires. Qual Life Res 1997, 6(3):237-247.

10. Herdman M, Fox-Rushby J, Badia X: A model of equivalence in the cultural adaptation of HRQoL instruments: the universalist approach. Qual Life Res 1998, 7(4):323-335.

II. Sperber AD: Translation and validation of study instruments for cross-cultural research. Gastroenterology 2004, I 26(I Suppl I):SI 24- I 28

12. Streiner DL, Norman GR: Health measurement scales: a practical guide to their development and use. 3rd edition. Oxford: Oxfor University Press; 2003.

13. Steiner M, Streiner DL: Validation of a revised visual analog scale for premenstrual mood symptoms: results from prospective and retrospective trials. Can J Psychiatry 2005, 50(6):327-332.

14. Bartko J): The intraclass correlation coefficient as a measure of reliability. Psychological reports 1966, I9(I):3-I I.

15. Wilson KA, Dowling AJ, Abdolell M, Tannock IF: Perception of quality of life by patients, partners and treating physicians. Qual Life Res 2000, 9(9): I04I-1052.

16. Landis JR, Koch GG: The measurement of observer agreement for categorical data. Biometrics 1977, 33(I): 159-174.

17. Cronbach LJ: Coefficient alpha and the internal structure of tests. Psychometrika 195I, I 6:297-334.

18. Nunnally JC, Bernstein IR: Psychometric theory. 3rd edition. New York: McGraw-Hill; 1994.

19. Ortiz MC, de Lima RA: Experiences of families with children and adolescents after completing a cancer treatment: support for the nursing care. Revista latino-americana de enfermagem 2007, I5(3):4II-4I7.

20. Cohen J: Statistical power analysis for the behavioral sciences. 2nd edition. Hillsdale: Lawrence Erlbaum; 1988.

21. Houtzager BA, Grootenhuis MA, Hoekstra-Weebers JE, Last BF: One month after diagnosis: quality of life, coping and previous functioning in siblings of children with cancer. Child: care, health and development 2005, 3 I (I):75-87.

22. McCaffrey $\mathrm{CN}$ : Major stressors and their effects on the wellbeing of children with cancer. J Pediatr Nurs 2006, 2 I (I):59-66.
23. Fleck MP, Louzada S, Xavier M, Chachamovich E, Vieira G, Santos L, Pinzon V: [Application of the Portuguese version of the instrument for the assessment of quality of life of the World Health Organization (WHOQOL-100)]. Rev Saude Publica 1999, 33(2): 198-205

24. Guyatt GH, Feeny DH, Patrick DL: Measuring health-related quality of life. Ann Intern Med 1993, I I 8(8):622-629.

25. Scarpelli AC, Paiva SM, Pordeus IA, Ramos-Jorge ML, Varni JW, Allison PJ: Measurement properties of the Brazilian version of the Pediatric Quality of Life Inventory (PedsQL) cancer module scale. Health and quality of life outcomes 2008, 6(I):7.

26. Allison PJ: Health-related quality of life comparisons in French and English-speaking populations. Community Dent Health 200I, I 8(4):2|4-2|8.

27. PedsQL ${ }^{\mathrm{TM}}$ Translation methodology [http://www.pedsql.org/ index.html]

28. Jenkinson C: Evaluating the efficacy of medical treatment: possibilities and limitations. Social science \& medicine (I 982) 1995 , 4 I ( I 0): | 395-| 40 |.

29. Shrout PE: Reliability. In Textbook in psychiatry epidemiology Edited by: Zahner TTA. New York: Wiley-Liss; 1995:2 I3-227.

30. Varni JW, Burwinkle TM, Katz ER, Meeske K, Dickinson P: The PedsQL in pediatric cancer: reliability and validity of the Pediatric Quality of Life Inventory Generic Core Scales, Multidimensional Fatigue Scale, and Cancer Module. Cancer 2002, 94(7):2090-2106.

31. Straus MA, Gelles JR: Physical violence in American families: risk factors and adaptations to violence in 8,145 families. New Brunswick: Transaction Publishers; 1995.

32. Li TC, Lin CC, Liu CS, Li Cl, Lee YD: Validation of the Chinese version of the diabetes impact measurement scales amongst people suffering from diabetes. Qual Life Res 2006, I5(10): 1613-1619.

33. Brabo EP, Paschoal ME, Biasoli I, Nogueira FE, Gomes MC, Gomes IP, Martins LC, Spector N: Brazilian version of the QLQ-LCI 3 lung cancer module of the European Organization for Research and Treatment of Cancer: preliminary reliability and validity report. Qual Life Res 2006, I5(9):I519-I524.

34. Upton P, Eiser C, Cheung I, Hutchings HA, Jenney M, Maddocks A, Russell IT, Williams JG: Measurement properties of the UKEnglish version of the Pediatric Quality of Life Inventory 4.0 (PedsQL) generic core scales. Health and quality of life outcomes 2005, 3:22.

35. Patenaude AF, Kupst MJ: Psychosocial functioning in pediatric cancer. Journal of pediatric psychology 2005, 30(I):9-27.

36. Weinberger M, Oddone EZ, Samsa GP, Landsman PB: Are healthrelated quality-of-life measures affected by the mode of administration? Journal of clinical epidemiology 1996, 49(2): | 35- | 40.

37. Goursand D, Paiva SM, Zarzar PM, Ramos-Jorge ML, Cornacchia GM, Pordeus IA, Allison PJ: Cross-cultural adaptation of the Child Perceptions Questionnaire I I-I4 (CPQ I I- I 4) for the Brazilian Portuguese language. Health and quality of life outcomes 2008 , 6:2.

Publish with Bio Med Central and every scientist can read your work free of charge

"BioMed Central will be the most significant development for disseminating the results of biomedical research in our lifetime. "

Sir Paul Nurse, Cancer Research UK

Your research papers will be:

- available free of charge to the entire biomedical community

- peer reviewed and published immediately upon acceptance

- cited in PubMed and archived on PubMed Central

- yours - you keep the copyright

BioMedcentral 